\title{
MECP2 mutations in Danish patients with Rett syndrome: High frequency of mutations but no consistent correlations with clinical severity or with the $\mathrm{X}$ chromosome inactivation pattern
}

\author{
Jytte Bieber Nielsen ${ }^{* 1,3}$, Karen Friis Henriksen ${ }^{2}$, Claus Hansen ${ }^{2}$, Asli Silahtaroglu ${ }^{2}$, \\ Marianne Schwartz ${ }^{1}$ and Niels Tommerup ${ }^{2}$
}

${ }^{1}$ Department of Clinical Genetics, Rigshospitalet University Hospital, University of Copenhagen, Blegdamsvej 9, DK-2100 Copenhagen Ø, Denmark; ${ }^{2}$ Department of Medical Genetics, IMBG, The Panum Institute, University of Copenhagen, Blegdamsvej 3, DK-2200 Copenhagen N, Denmark; ${ }^{3}$ The John F. Kennedy Institute, Gl. Landevej 7, DK-2600 Glostrup, Denmark

Rett syndrome (RTT) is a neurodevelopmental disorder, which almost exclusively affects girls, who, after an initial period of apparently normal development, display gradual loss of speech and purposeful hand use, gait abnormalities and stereotypical hand movements. In the year 2000, mutations in the gene for the methyl CpG binding protein 2, MECP2, have been identified in $35-80 \%$ of the patients in three different studies. We have identified 15 different MECP2 mutations in 26 of 30 Danish RTT patients. The mutations included five novel mutations (one point mutation, three smaller deletions involving identical regions in the gene, and one duplication). In contrast to the point mutations and the duplication, which all affect the methyl binding domain or the transcriptional repressing domain, the three overlapping deletions are clustered in the $3^{\prime}$ end of the gene. We found no consistent correlation between the type of mutation and the clinical presentation of the patient or the $\mathrm{X}$-inactivation pattern in peripheral blood. Our high mutation detection rate, compared to two of the previous studies, underscores the importance of the inclusion criteria of the patients and supports that MECP2 is the most important, if not the only, gene responsible for RTT. European Journal of Human Genetics (2001) 9, 178-184.

Keywords: Rett syndrome; mutations; MECP2; X-inactivation

\section{Introduction}

Rett syndrome, RTT, is a neurodevelopmental disorder, which almost exclusively affects girls. After a period for up to 18 months of apparently normal development, the affected girls suffer from gradual loss of speech and purposeful hand use, gait abnormalities and stereotypical hand movements. ${ }^{1}$ Based on clinical criteria ${ }^{2}$ the diagnosis

*Correspondence: Jytte Bieber Nielsen, Department of Clinical Genetics, 4062 Rigshospilalet University Hospital, University of Copenhagen, Blegdansvej 9, DK-2100 Copenhagen Ø, Denmark. E-mail: bieber@rh.dk Received 29 May 2000; revised 12 October 2000; accepted 20 October 2000 can not be made earlier than at about 2 years of age. The vast majority of cases are sporadic. However, the predominance of female patients suggested an X-linked dominant disorder, and polymorphic marker typing of the rare familial cases pointed to a RTT locus at Xq28. ${ }^{3-6}$ In addition X chromosome inactivation (XCI) studies $^{7,8}$ have shown a tendency towards skewed XCI with preferential inactivation of the paternal X chromosome.

By systematic mutation analysis of candidate genes in the Xq28 region, Amir et $a l^{9}$ detected mutations in the gene for the methyl CpG binding protein 2, MECP2. Shortly thereafter a survey of mutations found in North American patients was published. ${ }^{10}$ A mutation was found in $50 \%$ of the patients. 
Recently, a Swedish, ${ }^{11}$ a British, ${ }^{12}$ a French ${ }^{13}$ and a German ${ }^{14}$ study with mutations in MECP2 in $35-80 \%$ of the patients have been published and the North American study has been updated. $^{15}$

The gene product $\mathrm{MeCP} 2$, which was initially identified based on the selective binding to methylated DNA, ${ }^{16}$ belongs to a group of proteins with a capacity to bind to CpG pairs in promoter regions of genes whereby transcription is repressed. The gene covers about $70 \mathrm{~kb}$, of which the protein coding regions constitute 1458 base pairs distributed over three exons. It encodes a protein of 486 amino acids, which contains a methyl binding domain, MBD, of 85 amino acids, a transcriptional repression domain, TRD, of 104 amino acids ${ }^{17}$ and a corepressor-interacting region in between. ${ }^{18}$

We have identified 69 RTT patients in Denmark, born in the years 1923 to 1997, including 61 classical cases and eight variants. We have tested the first 30 of these patients for mutation in the coding region, a part of the $5^{\prime}$ UTR and a part of the 3' UTR of MECP2 and correlated the mutation spectrum with the XCI. In three of the four cases where we did not find mutations, we excluded gross deletions of MECP2 by fluorescence in situ hybridization (FISH).

\section{Materials and methods}

\section{Identification and classification of patients}

The patient group consisted of 30 sporadic cases born in the years 1960 to 1994 . These patients were chosen out of the 69 known cases because of the availability of DNA samples both from the patients and their parents. Twentynine of the patients were Danish and one was a Romanian immigrant. All the patients were investigated clinically by one of us (JBN) in the period 1986-1997. They were classified using the criteria established by the Rett syndrome Diagnostic Work group. ${ }^{2}$ Twenty-seven of the patients were diagnosed with classical RTT and three as forme fruste (FF) variants with a milder phenotype. ${ }^{19}$ Furthermore the patients were evaluated by a scoring system with respect to: (1) hand function; (2) eye contact; and (3) gait function (Table 2).

\section{DNA samples}

DNA was isolated from either blood or cultivated fibroblasts by routine methods. The DNA had been stored at $-20^{\circ} \mathrm{C}$ for up to 10 years before analysis.

\section{Mutation detection}

The two independent laboratories at Rigshospitalet and the Panum Institute carried out the mutation detection using methods (a) and (b), respectively, as described below. In both laboratories genomic DNA was amplified into eight fragments using the eight primer pairs covering the coding sequences of MECP2 and conditions described by Amir et al ${ }^{9}$ with some modifications stated by Wan et al. ${ }^{10}$ The $5^{\prime}$ untranslated region, 5' UTR, was included by primer pair 1 . (We are aware that our sequencing does not cover the newly discovered exon upstream of exon $1^{20}$ ). Additionally the first $\mathrm{kb}$ of the 3' untranslated region, 3' UTR, was sequenced, using the following four primer sets for the PCR reaction and the annealing temperature $60^{\circ} \mathrm{C}$ : The first set of UTR primers overlapped with the last set of exon primers (+numbers indicate primer location relative to the stop codon): +140Forward: 5'-GTTTCGTGGCTTCAGGGTGGGAGTA-3' +429Reverse: 5'-GGGAGCTGTAGACGGGGCACTG-3' +322Forward: 5'-CCTCCCGCCCAAACCT-3' +613Reverse: 5'GTGAAGCGTCCTTGCCAGTT-3' +552Forward: 5' CCGTGGTGGCTCAAGTCTGG-3' +850Reverse: 5' CACCCCGCTGTGCTCTGC-3' +785Forward: 5'-GAGGCAGTAGGACAAGGTG-3' +1145Reverse: 5'-TATGTGCTGAGCCCACTTTA-3'. The PCR products from the coding sequences were examined for possible mutations by two different methods of direct sequencing, both utilising the primers used for PCR amplification as sequencing primers: (a) Direct sequencing of purified PCR products by use of fluorescentdye terminators (BigDye ${ }^{\mathrm{TM}}$ Terminator Cycle Sequencing from PE Biosystems). The products were analysed on an ABI 310 automatic sequencer. (b) Direct sequencing of purified PCR using cycle sequencing with Thermosequenase and 33Plabelled dideoxynucleotides (Amersham Pharmacia Biotech) followed by electrophoresis on $6 \%$ polyacrylamide gels and autoradiography. All mutations were identified by both methods. The PCR products from the 3' UTR were sequenced by method (b) only.

\section{Deletion analysis by fluorescence in situ hybridisation (FISH)}

Three of the four patients without identified mutations were available for further analysis by FISH to exclude possible larger deletions of the RTT region in Xq28. The PAC clone containing the MECP2 gene, 671_d_9, was purchased from Research Genetics, Inc and used for FISH analysis. Labelling of the probe, fluorescence in situ hybridisation and detection were carried out as described previously. $^{21}$ For each experiment $200 \mathrm{ng}$ of biotin-16dUTP-labelled PAC DNA was used together with a digoxigenin-labelled centromere probe specific for the $\mathrm{X}$ chromosome. The FISH signals were visualised with a Leica DMRB epifluorescence microscope equipped with a Sensys 1400 CCD camera (Photometrics, USA) and IPLab Spectrum Imaging Software (Vysis, USA).

\section{$\mathrm{X}$ chromosome inactivation}

XCI was assessed by the method described by Allen et al. ${ }^{22}$ In brief, DNA from the patient was amplified by primers flanking the CAG repeat region of exon 1 in the androgen receptor gene. The PCR products were analysed using ABI310 (PE-Biosystems). The allele size and peak area were determined before and after HhaI cleavage. 


\section{Results}

Using a traditional approach by PCR amplification of the coding sequences and parts of their flanking sequences, followed by scanning for mutations, we identified 15 different mutations in the MECP2 gene in 26 of the 30 patients, of which five were novel (Table 1). Except for two mutations they were all localised to exon 3 (Figure 1). None of the mutations were found in any of the parents.

\section{Novel mutations}

Truncating mutations In Patient 29 with classical RTT and random $\mathrm{XCI}$, we identified a single nucleotide deletion, 76delC, resulting in a truncated protein of 31 amino acids, which is predicted to encode a polypeptide lacking both MBD and TRD.

In Patient 18 with classical RTT and a totally skewed XCI sequencing showed that the patient harboured a duplication of $14 \mathrm{bp}$ (bp 766-779). The mutation is predicted to introduce a stop codon downstream after 32 missense amino acids leading to a truncated protein of 292 amino acids missing part of the TRD domain.

By PCR using primer pairs 7 we observed PCR products from three patients (Patients 1, 2 and 15), two classical and one FF, having a smaller size in addition to the expected one. Sequencing these products revealed three deletions of 50, 44 and $26 \mathrm{bp}$, respectively. All three deletions are out of frame and will introduce identical new stop codons, resulting in truncated proteins ending upstream of the TRD. Interestingly, the deletions involve the same region of the gene, starting at nucleotides 1144, 1156 and 1163, respectively. This type of deletion of more than one bp has been reported before in $\mathrm{RTT}^{11-15}$ and seems to represent a recurrent event. The two patients with 26 and $50 \mathrm{bp}$ deletions showed a random XCI, whereas the patient with the $44 \mathrm{bp}$ deletion had a slightly skewed XCI $(81 \% / 19 \%)$. The patient with the smallest deletion has a milder variant phenotype.

\section{Previously reported mutations}

Y141X was found in two patients (Patients 13 and 16) both with classical RTT. This $423 \mathrm{C} \rightarrow \mathrm{G}$ transversion changes tyrosine codon 141 in exon 3 to a stop codon. This is one of the few mutations not involving a CpG hotspot. One of the patients showed a skewed XCI pattern, while the other is classified as random. This mutation is expected to result in a very truncated protein, which must be expected to be rapidly degraded. This mutation has only been found in one more study. $^{15}$

We identified four patients with the mutation R255X, three patients with R168X, and three patients with R294X. The mutation $808 \mathrm{C} \rightarrow \mathrm{T}, \mathrm{R} 270 \mathrm{X}$, was found in a single patient with classical RTT. The mutation leads to a truncated protein, albeit not very shortened, which may retain its $\mathrm{mC}$ binding capacity. The patient showed a $100 \%$ skewed XCI pattern in leucocytes.

Furthermore we found one patient with R106W, three patients with T158M, one with P302L and two with R306C.

The patient with the R106W mutation had a healthy twin sister, who did not have the mutation. DNA analysis with polymorphic markers disclosed dizygosity of the twins.

\section{Silent mutations}

In Patient 8, who had an R255X mutation, we identified a $\mathrm{C} \rightarrow \mathrm{T}$ transition at nucleotide 582 . This variation $\mathrm{S} 194 \mathrm{~S}$ was inherited from the father, and thus probably represents a rare

Table 1 Patients and the mutations of MECP2 found in this study*

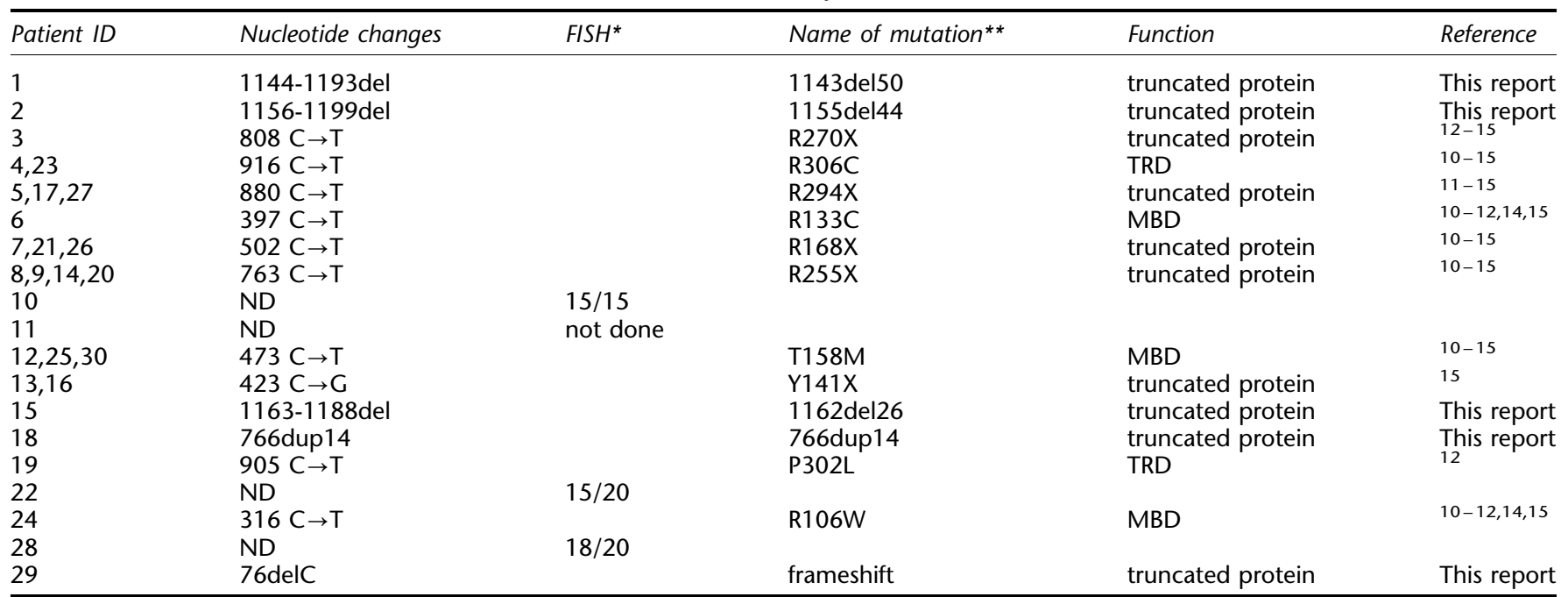

*Proportion of cells with FISH signals on both X chromosomes. ${ }^{* *}$ Nomenclature according to Antonarakis et al. ${ }^{25}$ MBD: methyl binding domain; TRD: transcriptional repression domain. ND: not detected. 


\section{MUTATIONS OF $\boldsymbol{M E C P 2}$ IN DANISH PATIENTS WITH RETT SYNDROME}

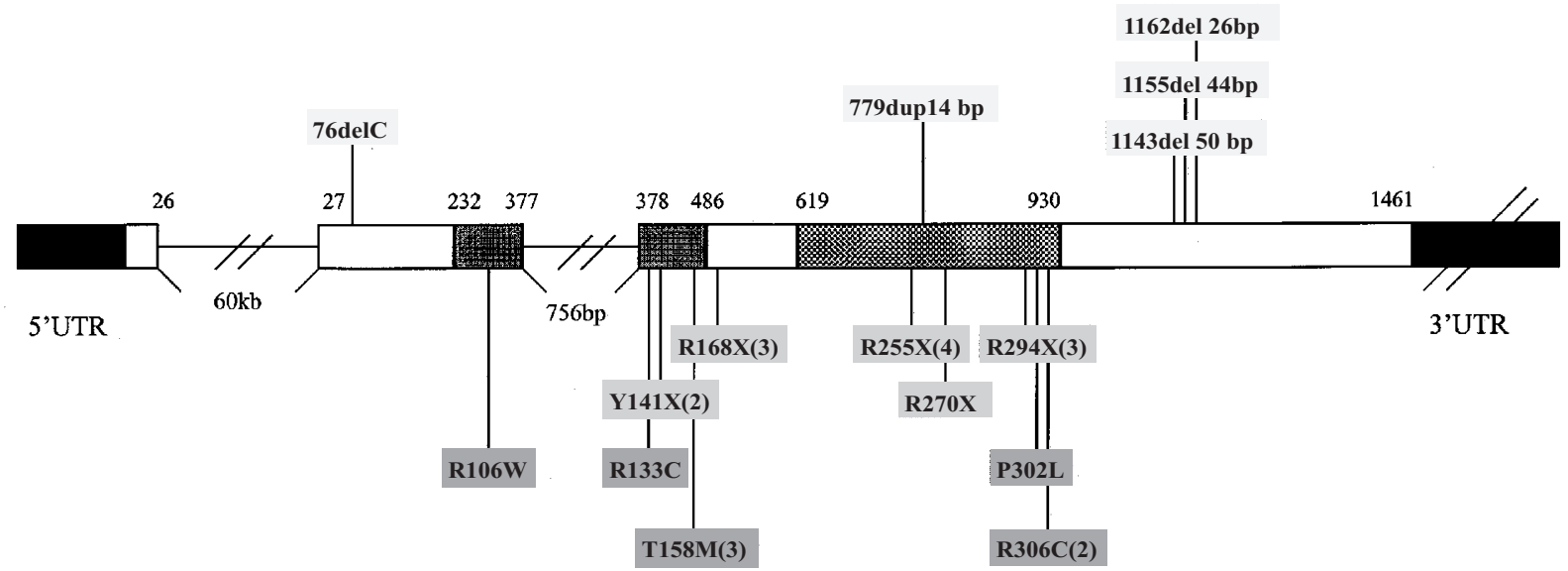

MBD

\section{FRAMESHIFT MUTATION NONSENSE MUTATION MISSENSE MUTATION}

Figure 1 All the Danish mutations found in the present study are shown in the figure. The numbers in parentheses after the name of the mutation indicates the number of patients with the mutation in question. When a number is not given, there has only been one patient with the mutation. Nomenclature according to Antonarakis et al. ${ }^{25} \mathrm{MBD}$ : methyl binding domain; TRD: transcriptional repression domain; UTR: untranslated region.

polymorphism. The location of the mutations is shown in Figure 1.

\section{Deletion analysis by fluorescence in situ hybridisation (FISH)}

Except for Patient 29 who carries a de novo inversion of chromosome $2(46, X X, \operatorname{inv}(2)(p 21 q 11))$, all patients have a normal female karyotype $(46, \mathrm{XX})$. The 671 d_9 FISH probe gave signals on the distal Xq-region on both $\mathrm{X}$ chromosomes in $75-100 \%$ of the metaphases from both normal control females, and from the three available RTT patients (Patients 10, 22 and 28) in whom we did not detect a mutation by molecular analysis (Table 1 ).

\section{$\mathrm{X}$-inactivation}

Twenty-eight of the families were informative. Eighteen patients had random XCI pattern, defined as inactivation ratios between 21 and 79 . Of the nine patients (30\%) with skewed XCI, five had a totally skewed pattern in blood. In eight patients the paternal $\mathrm{X}$ chromosome was preferentially inactivated.

\section{Evaluation by severity score}

We have evaluated all our patients by a scoring system with respect to: (1) hand function; (2) eye contact; and (3) gait function (Table 2). We did not find any difference in severity score between the truncating mutations and the missense mutations.

\section{Discussion}

We have identified a mutation in $26 / 30$ patients $(87 \%)$ with RTT, of which five were novel. All the mutations were de novo. The mutational spectrum mimics recently published mutations, but in addition we have extended the mutation analysis to the first part of the 3' UTR without detecting any mutation. Gross rearrangements in those patients without detectable mutations were excluded by FISH analysis. Thus, all the mutations occurred in the MECP2 coding region, and the majority were found in the MBD or TRD. Our detection rate is so far the highest published compared to the $35 \%$ by Xiang et al, ${ }^{11}$ the $50 \%$ by Wan et al ${ }^{10}$ and the $80 \%$ recently reported by Cheadle et al. ${ }^{12}$ The latter group explain their high detection was due to the 'long-read sequence analysis' method used. Since we have used a traditional approach as reported by others ${ }^{9,10}$ and have identified even more mutations than any other group, we believe that the inclusion criteria must be the most important factor in relation to the detection rate.

Our results confirm the previous observations ${ }^{10-15}$ that almost all point mutations in $M E C P 2$ are $\mathrm{C} \rightarrow \mathrm{T}$ transitions, all at $\mathrm{CpG}$ dinucleotides. In our patient group, 19 of the 26 
Table 2 Genotype-phenotype correlation

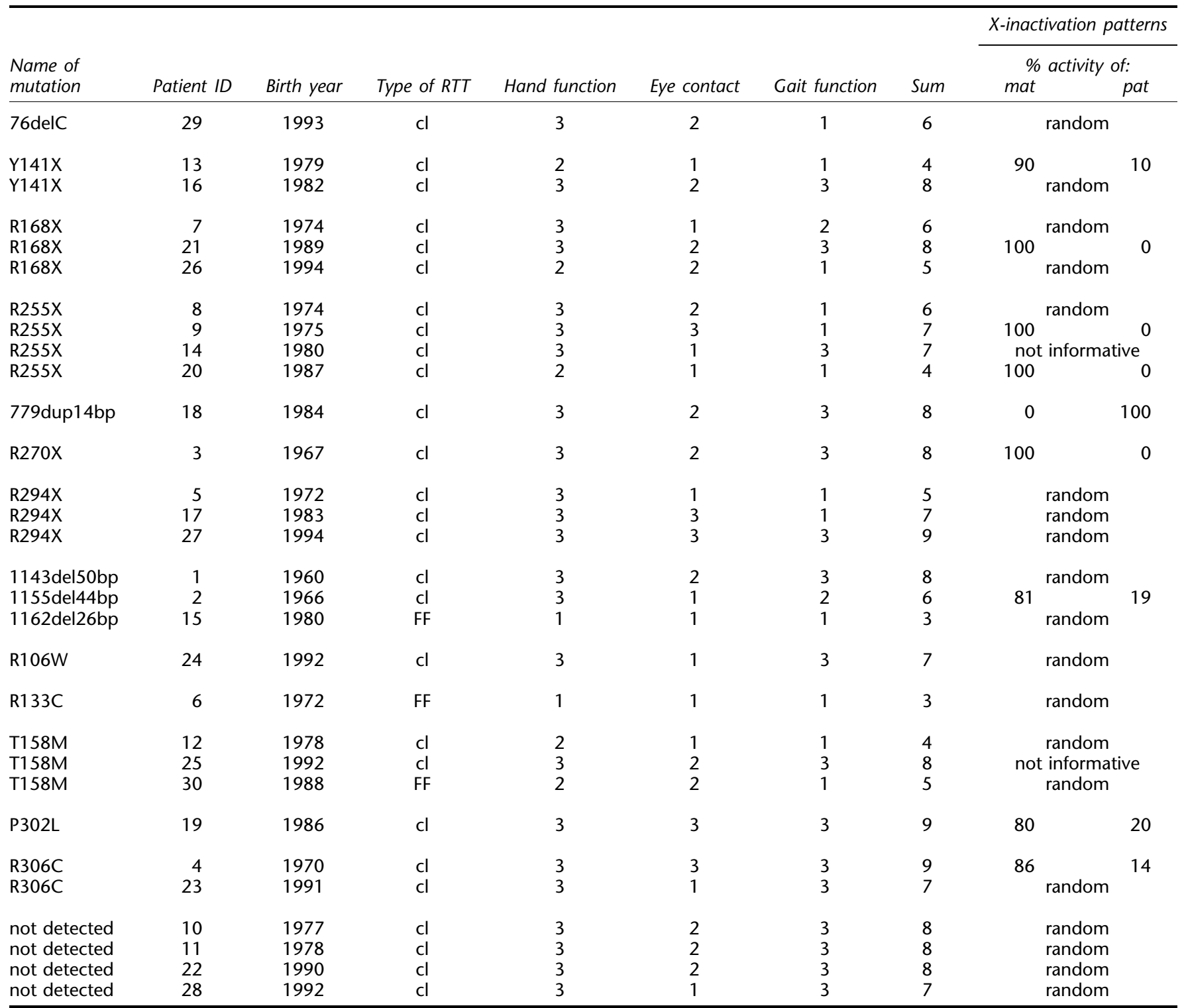

Hand function: (1) can hold a glass, can use a spoon or fork; (2) can sometimes grasp an object; (3) does never use her hands purposefully. Eye contact: (1) intense, use eye pointing; (2) direct eye contact possible to obtain; (3) eye contact very difficult to obtain. Gait function: (1) walks independently; (2) has lost gait function; (3) has never walked.

mat: maternal, pat: paternal.

detected mutations belong to this type of transition, underscoring the high mutation rate of this dinucleotide. ${ }^{23}$ Another striking feature is the recurrent smaller deletions upstream of the TRD in exon 3 . We identified three deletions of 50, 44 and 26 bases, respectively. Recent studies have reported a total of 17 deletions in this part of the gene. ${ }^{11-15}$ None of the total of 20 deletions are identical. All our deletions create frameshifts and introduce a stop codon at identical positions with respect to cDNA. Hence, the resulting predicted truncated proteins are almost of the same size in all these patients. Although no direct repeats were obvious, the deletion breakpoints are flanked by several repeated sequences and might thus be the result of slipped mispairing.

We were unable to demonstrate mutations in MECP2 in only four of these 30 unrelated, randomly selected Danish patients with RTT. All four cases were typical classical RTT. PCR-based mutation detection will not reveal larger deletions. However, in three of these cases we could exclude the presence of a larger deletion of MECP 2 by 
FISH. In the studies of Wan et $a l^{10}$ and Xiang et al ${ }^{11}$ it was not possible to directly compare the clinical features with the mutational spectrum in MECP2. However, in the recent study of Cheadle et $a{ }^{12}{ }^{12}$ where they used a more detailed clinical scoring system to assess the severity of the disease, a significantly higher score was reported in patients harbouring truncating mutations. In an attempt to compare our study with that of Cheadle et $a l,{ }^{12}$ we have evaluated all our patients by a scoring system with respect to: (1) hand function: (2) eye contact; and (3) gait function (Table 2). We found patients with both severe and milder phenotypes in both groups (Table 2): In the group with missense mutations (eight patients), two patients had the FF variant with preserved gait function, and six patients had classical RTT. Five of these had never had gait function and one is still ambulant. In the group with truncating mutations (18 patients), there was one with the FF variant (who is still ambulant) and 17 with classical RTT (of which seven have never walked, two have lost ambulation and eight have preserved this ability). Thus, we were not able to confirm any correlation between mutation type or position with the scores. Furthermore, the XCI pattern did not seem to change the score: Of the four patients harbouring the R255X mutation, two have completely skewed XCI - they both have classical RTT, but neither of them is extremely mildly or extremely severely affected. As long as nothing is known concerning the fate of the encoded protein, it is not possible to make any conclusion about the presence or lack of genotype-phenotype correlation. Although we are aware that our study is too small for statistical evaluation, all our patients were evaluated by the same physician, assuring a uniform use of the diagnostic criteria. In the recent study by Amir et $a l^{15}$ a thorough clinical evaluation was made which supports our finding of lack of correlation between genotype and phenotype. Even though they found positive correlation between truncating mutations and breathing abnormalities and low levels of the neurotransmitter metabolite homovanillic acid in spinal fluid, the major clinical features of RTT and the degree of severity appeared to be independent of mutation type and mutation position.

One patient in the study of Wan et $a l^{10}$ had both RTT and features of incontinentia pigmenti (IP). At that time the gene for IP was not yet identified but was known to be located at Xq28. Since the patient carried a frameshift mutation resulting in a truncated protein of only 138 amino acids, it was speculated that this mutation, which was the closest to a loss-of-function mutation identified so far, might be associated with a more complex phenotype including IP. However, our study revealed one mutation predicting a truncated protein of only 31 amino acids in an ambulatory patient with classical RTT. In addition this patient also carries a de novo pericentric inversion of chromosome 2 (inv(2)(p21q11)). She has random XCI $(62 \% / 38 \%)$ and is not more severely affected than one of the patients with a deletion in the $3^{\prime}$ end of the gene. The random XCI contradicts the conclusion by Amir et al ${ }^{15}$ that their two patients with the early truncating mutations Y141X and 411delG, survive because of their non-random pattern of XCI, with the normal allele being activated in most cells. The mutation resulting in the most truncated protein reported so far was found in a patient from an autism clinic with a relatively mild phenotype - a Q19X mutation with a predicted gene product consisting of only 19 amino acids. ${ }^{24}$ Although the XCI was not examined in this patient, these two observations strengthen the conclusion that mutations leading to premature truncation at the beginning of the protein do not necessarily give rise to a more severe phenotype than other mutations in MECP2.

The coding sequence of $M E C P 2$ contains 35 CpG dinucleotides with the possibility of $\mathrm{C} \rightarrow \mathrm{T}$ transitions that would lead to missense or nonsense mutations. Of these, six CpG pairs are in exon 3 in the region covered by primer pair 8 , where neither others nor we have detected any mutations. In exon 1 there are no CpG pairs, which in part could explain that no mutation so far has been found in exon 1. One could also suspect that mutations in these regions are either 'silent' or lead to a phenotype different from RTT.

The frequency of the individual mutations was slightly different in the four studies. The most common mutation in our study was the R255X, which we found in four of 26 (15\%) patients. So far the R168X is the most common mutation found, ranging from 12 to $39 \%$ of all mutations found in the studies. The position of all the mutations is shown in Figure 1.

XCI studies performed on peripheral lymphocytes have shown a tendency towards skewed XCI with preferential inactivation of the paternal $\mathrm{X}$ chromosome. ${ }^{7,8}$ However, XCI did not give the full explanation for the clinical differences between patients with identical mutations. For example, the three patients with similar deletions were phenotypically quite different: Patient 15 with FF had random XCI as had Patient 1 who had the classical form and never had had gait function. None of the five patients with totally skewed XCI got a clinical score higher than 8, 9 being the most severe clinical condition. Yet, the three patients with a score of 8 are severely affected, while the two other patients have a much better motoric function. An obligate female carrier, presenting with an almost normal phenotype and a mutation in MECP2, had a 95\%/ $5 \%$ skewed XCI in blood. ${ }^{4,6,10}$ None of our patients with a $100 \% / 0 \%$ skewed XCI in blood cells were that mildly affected. Although we could not relate the XCI pattern to the phenotype, it is still conceivable that the consequence of a mutation may be modified by the X-inactivation pattern. First of all, the XCI pattern may be different in other, more relevant tissues, such as the brain. Also, it is possible that analysis of XCI patterns could be useful if it was known whether the mutated or the normal $X$ chromosome was preferentially inactivated. 
9 Amir RE, Van den Veyver IB, Wan M, Tran CQ, Francke U, Zoghbi HY: Rett syndrome is caused by mutations in X-linked MECP2, encoding methyl-CpG-binding protein. Nature Genet 1999; 23: $185-188$.

10 Wan M, Lee SSJ, Zhang X et al: Rett syndrome and beyond: Recurrent spontaneous and familial MeCP2 mutations at CpG hotspots. Am J Hum Genet 1999; 65: 1520-1529.

11 Xiang F, Buervenich S, Nicolao P, Bailey ME, Zhang Z, Anvret M: Mutation screening in Rett syndrome patients. J Med Genet 2000; 37: 250-255.

12 Cheadle JP, Gill H, Fleming N et al: Long-read sequence analysis of the MECP2 gene in Rett syndrome patients: correlation of disease severity with mutation type and location. Hum Mol Genet 2000; 9: 1119-1129.

13 Bienvenu T, Carrie A, de Roux N et al: MECP2 mutations account for most cases of typical forms of Rett syndrome. Hum Mol Genet 2000; 9: $1377-1384$

14 Huppke P, Laccone F, Kramer N, Engel W, Hanefeld F: Rett syndrome: analysis of MECP2 and clinical characterization of 31 patients. Hum Mol Genet 2000; 9: 1369-1375.

15 Amir RE, Van den Veyver IB, Schultz R et al: Influence of mutation type and $\mathrm{X}$ chromosome inactivation on Rett syndrome phenotypes. Ann Neurol 2000; 47: 670-679.

16 Meehan RR, Lewis JD, Bird AP: Characterization of MeCP2, a vertebrate DNA binding protein with affinity for methylated DNA. Nucleic Acids Res 1992; 20: 5085 - 5092.

17 D'Esposito M, Quaderi NA, Ciccodicola A et al. Isolation, physical mapping, and northern analysis of the X-linked human gene encoding methyl CpG-binding protein, MECP2. Mamm Genome 1996; 7: 533-535.

18 Nan X, Ng HH, Johnson CA et al: Transcriptional repression by the methyl-CpG-binding protein MeCP2 involves a histone deacetylase complex. Nature 1998; 393: 386-389.

19 Hagberg BA, Skjeldal OH: Rett variants: a suggested model for inclusion criteria. Pediatr Neurol 1994; 11: 5-11.

20 Reichwald $\mathrm{K}$, Thiesen J, Wiehe T et al: Comparative sequence analysis of the MECP2-locus in human and mouse reveals new transcribed regions. Mamm Genome 2000; 11: 182-190.

21 Silahtaroglu A, Hol FA, Jensen PK et al: Molecular cytogenetic detection of $9 \mathrm{q} 34$ breakpoints associated with nail patella syndrome. Eur J Hum Genet 1999; 7: 68-76.

22 Allen RC, Zoghbi HY, Moseley AB, Rosenblatt HM, Belmont JW: Methylation of HpaII and HhaI sites near the polymorphic CAG repeat in the human androgen-receptor gene correlates with $\mathrm{X}$ chromosome inactivation. Am J Hum Genet 1992; 51: 1229 1239.

23 Krawczak M, Ball EV Cooper DN: Neighbouring-nucleotide effects on the rate of germ-line single-base-pair substitution in human genes. Am J Hum Genet 1998; 63: 474-488.

24 Kim SJ, Cook Jr EH: Novel de novo nonsense mutation of MECP2 in a patient with Rett syndrome. Hum Mutat 2000; 15: $382-383$.

25 Antonarakis SE: Recommendations for a nomenclature system for human gene mutations. Nomenclature Working Group. Hum Mutat 1998; 11: 1-3. 